ARTICLE

https://doi.org/10.1038/s41467-019-13145-x

\title{
Genetic variants of calcium and vitamin D metabolism in kidney stone disease
}

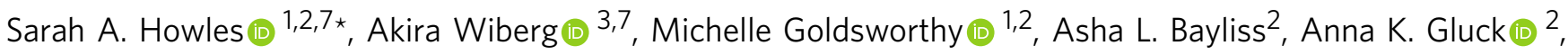
Michael Ng $\mathbb{D}^{3}{ }^{3}$, Emily Grout ${ }^{1}$, Chizu Tanikawa ${ }^{4}$, Yoichiro Kamatani [i] ${ }^{5}$, Chikashi Terao (i) ${ }^{5}$, Atsushi Takahashi (10 ${ }^{5}$, Michiaki Kubo ${ }^{5}$, Koichi Matsuda (i) ${ }^{6}$, Rajesh V. Thakker (1) ${ }^{2}$, Benjamin W. Turney ${ }^{1} \&$ Dominic Furniss (iD ${ }^{3}$

Kidney stone disease (nephrolithiasis) is a major clinical and economic health burden with a heritability of $\sim 45-60 \%$. We present genome-wide association studies in British and Japanese populations and a trans-ethnic meta-analysis that include 12,123 cases and 417,378 controls, and identify 20 nephrolithiasis-associated loci, seven of which are previously unreported. A CYP24A1 locus is predicted to affect vitamin D metabolism and five loci, DGKD, $D G K H, W D R 72, G P I C 1$, and $B C R$, are predicted to influence calcium-sensing receptor (CaSR) signaling. In a validation cohort of only nephrolithiasis patients, the CYP24A1-associated locus correlates with serum calcium concentration and a number of nephrolithiasis episodes while the DGKD-associated locus correlates with urinary calcium excretion. In vitro, DGKD knockdown impairs CaSR-signal transduction, an effect rectified with the calcimimetic cinacalcet. Our findings indicate that studies of genotype-guided precision-medicine approaches, including withholding vitamin D supplementation and targeting vitamin D activation or CaSRsignaling pathways in patients with recurrent kidney stones, are warranted.

\footnotetext{
${ }^{1}$ Nuffield Department of Surgical Sciences, University of Oxford, Oxford, UK. ${ }^{2}$ Academic Endocrine Unit, Radcliffe Department of Medicine, University of Oxford, Oxford, UK. ${ }^{3}$ Nuffield Department of Orthopaedics, Rheumatology and Musculoskeletal Sciences, University of Oxford, Oxford, UK. ${ }^{4}$ Laboratory of Genome Technology, Human Genome Centre, University of Tokyo, Tokyo, Japan. ${ }^{5}$ RIKEN Centre for Integrative Medical Sciences, Yokohama, Kanagawa, Japan. ${ }^{6}$ Laboratory of Clinical Genome Sequencing, Department of Computational Biology and Medical Sciences, University of Tokyo, Tokyo, Japan. ${ }^{7}$ These authors contributed equally: Sarah A. Howles, Akira Wiberg *email: sarah.howles@nds.ox.ac.uk
} 
K idney stones affect $\sim 20 \%$ of men and $\sim 10 \%$ of women by 70 years of age ${ }^{1}$ and commonly cause debilitating pain. The prevalence of this disorder is increasing and the United States is predicted to spend over $\$ 5$ billion per year by 2030 on its treatment ${ }^{2}$. Unfortunately, up to $50 \%$ of individuals will experience a second kidney stone episode within 10 years of their initial presentation ${ }^{3}$ and recurrent stone disease is linked to renal function decline $e^{4}$.

Twin studies have reported a heritability of $>45 \%$ and $>50 \%$ for stone disease and hypercalciuria, respectively ${ }^{5,6}$ and a strong family history of urolithiasis, including a parent and a sibling, results in a standard incidence ratio (SIR) for stone formation of $>50$ in contrast to a SIR of 1.29 in spouses ${ }^{7}$. Four genome-wide association studies of nephrolithiasis have been published identifying fifteen loci associated with disease ${ }^{8-11}$; however no transethnic studies have been undertaken.

To increase understanding of the common genetic factors contributing to risk of nephrolithiasis, we present a genome-wide association study (GWAS) using the UK Biobank resource ${ }^{12}$ and perform a subsequent meta-analysis with the summary statistics from the Biobank Japan nephrolithiasis genome-wide association study ${ }^{11,13}$ to identify 20 loci associated with nephrolithiasis. One such locus is associated with CYP24A1 and is predicted to affect vitamin $\mathrm{D}$ metabolism and five loci, DGKD, DGKH, WDR72, GPIC1, and $B C R$, are predicted to influence calcium-sensing receptor (CaSR) signaling. In a validation cohort of nephrolithiasis patients, we find that the CYP24A1-associated locus correlates with serum calcium concentration and number of kidney stone episodes and that the DGKD-associated locus correlates with urinary calcium excretion. Moreover, DGKD knockdown impairs CaSR-signal transduction in vitro, an effect that is rectified by the calcimimetic cinacalcet, thereby supporting the role of DGKD in CaSR signaling. Our findings suggest that further studies into the utility of genotyping to inform risk of incident kidney stone disease prior to vitamin $\mathrm{D}$ supplementation and to guide precision-medicine approaches, by targeting CaSRsignaling or vitamin $\mathrm{D}$ activation pathways in patients with recurrent kidney stones, are warranted.

\section{Results}

Association studies and in silico analyses. Genome-wide association studies were undertaken in UK and Japanese populations. Stone forming individuals were identified from the UK Biobank using ICD-10 and OPCS codes and a single UK Biobank selfreported operation code for kidney stone surgery and excluded if recorded to have a disorder known to predispose to kidney stone disease (Supplementary Table 1 and 2). In contrast, Biobank Japan cases were diagnosed and enrolled by physicians; information regarding conditions known to predispose to kidney stones was unavailable. UK Biobank genome-wide association analysis was undertaken across 547,011 genotyped SNPs and $\sim 8.4$ million imputed SNPs with MAF $\geq 0.01$ and Info Score $\geq 0.9$, using a linear mixed non-infinitesimal model implemented in BOLT-LMM v2.3 $3^{14}$. Biobank Japan GWAS was conducted using a logistic regression model incorporating age, sex, and the top 10 principal components as covariates. Subsequently, a trans-ethnic meta-analysis was performed using the summary statistics from the UK and Japanese GWAS data sets to integrate data from 12,123 stone formers and 417,378 controls.

Twenty genetic loci associating with nephrolithiasis were identified, 10 of which were initially identified from the UK Biobank discovery cohort (Supplementary Table 3) and another 10 from the subsequent trans-ethnic meta-analysis with Japanese GWAS summary statistics (Table 1, Fig. 1, and Supplementary Fig. 1 ${ }^{11}$. Seven of the identified loci have not previously been reported to associate with kidney stone disease at GWAS (Table 1) ${ }^{8-11,15,16}$. The allelic effects were concordant across both studies at all 20 loci, with minimal evidence of heterogeneity between the two GWAS at the majority of loci, with a Q-statistic $p$-value $>0.05$ at 17 out of 20 loci (Supplementary Table 4), suggesting that the genetic architecture of kidney stone disease is very similar between populations of European and East Asian ancestry. For the seven previously unreported loci, we found that the effects are directionally concordant in both datasets and of the same magnitude, with the exception of rs13054904 (the $B C R$ locus). This locus therefore highlights ethnic differences in the predisposition to renal stone disease.

Out of 849 SNPs with a meta-analysis significant $p$-value of $p<$ $5.0 \times 10^{-8}$, many demonstrated evidence of potential functionality: 33 SNPs had a combined annotation-dependent depletion (CADD) score $>12.37$, the threshold suggested for deleterious SNPs ${ }^{17}$. A further 54 SNPs had a RegulomeDB score of $2 b$ or higher, which is likely to affect protein binding ${ }^{18}$ (Supplementary Data 1).

Fifty-four candidate genes were identified via in silico analysis of these 20 loci based on FUMA positional mapping, functional annotation, and biological plausibility ${ }^{19}$. MAGMA gene-property analysis implemented in FUMA revealed a striking overexpression of these genes in the kidney cortex; the GENE2FUNC tool demonstrated enrichment for gene ontologies associated with transmembrane ion transport, renal function, and calcium homeostasis, including response to vitamin D (Supplementary Figs. $2-3$ and Supplementary Table 5) ${ }^{20}$.

CYP24A1 associated locus. We observed a genome-wide significant signal at a locus (rs17216707) that is $\sim 38 \mathrm{~kb}$ upstream of CYP24A1, a gene encoding cytochrome P450 family 24 subfamily A member 1 (CYP24A1), an enzyme that metabolizes active 1,25dihydroxyvitamin D to inactive 24,25 -dihydroxyvitamin D. Lossof-function mutations in CYP24A1 cause autosomal recessive infantile hypercalcaemia type 1 (OMIM 126065) ${ }^{21}$. We postulated that the CYP24A1 increased-risk allele associates with decreased CYP24A1 activity, leading to perturbations of calcium homeostasis and mimicking an attenuated form of infantile hypercalcaemia type 1 . Therefore, associations of rs17216707 with serum calcium, phosphate, parathyroid hormone (PTH), and 25hydroxyvitamin D concentrations, and urinary calcium excretion, and number of kidney stone episodes were sought in a validation cohort of 440 kidney stone formers attending the Oxford University Hospitals NHS Foundation Trust for treatment of kidney stones. Only stone forming patients were recruited, $15 \%$ of individuals were taking medications, including steroids and diuretics, that may affect risk of kidney stone formation. 1,25-hydroxyvitamin D levels were unavailable. Reference ranges for 24-hour urinary calcium excretion differ for men and women ${ }^{22}$, thus associations with urinary calcium excretion were examined separately.

Individuals homozygous for the CYP24A1 increased-risk allele rs17216707 (T) had a significantly increased mean serum calcium concentration when compared to heterozygotes (mean serum calcium $2.36 \mathrm{mmol} / \mathrm{l}$ (TT) vs. $2.32 \mathrm{mmol} / \mathrm{l}$ (TC); trend test p-value for additive effect $=0.023$ ) (Table 2). Analysis of the allelic frequencies of this SNP between cases and controls within the UK Biobank cohort was not able to provide definitive evidence for the recessive model over other genetic models (Supplementary Table 6). rs17216707 (T) homozygotes had more kidney stone episodes than heterozygotes (mean number of stone episodes 4.0 (TT) vs. 2.4 (TC), Mann-Whitney U-test $p=0.0003$ ) and there was a significant correlation across genotypes (TT vs. TC vs. CC) with number of stone episodes (Kruskal-Wallis $p=0.0024$ ) 


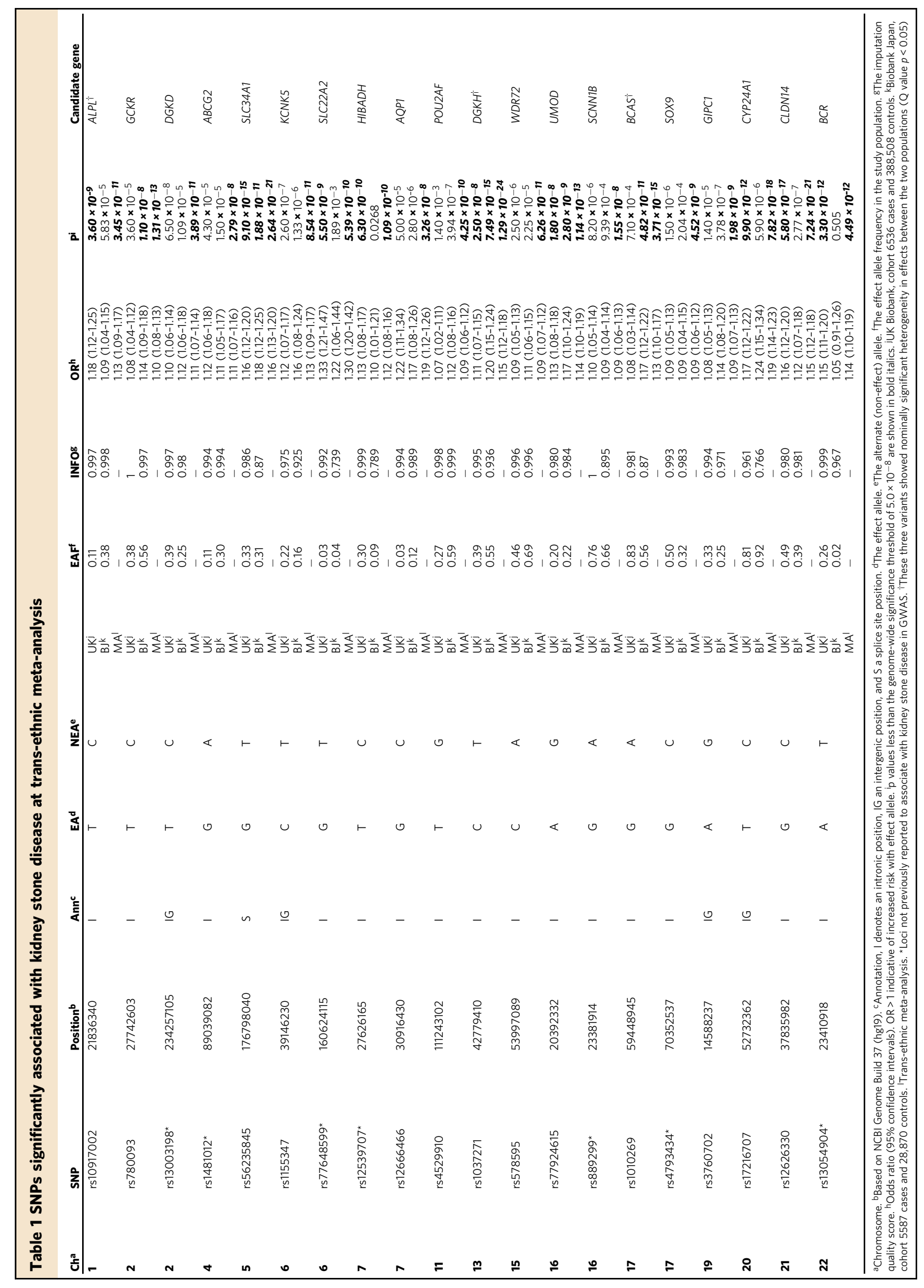



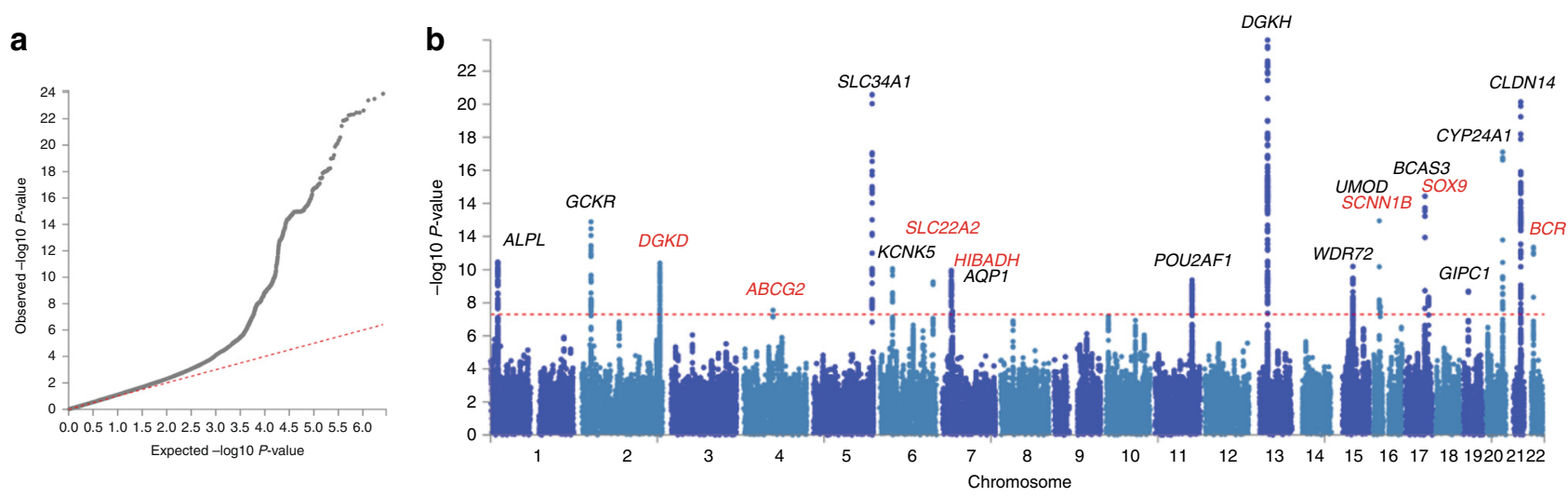

Fig. 1 Results of trans-ethnic genome-wide association study in kidney stone disease. A trans-ethnic meta-analysis of kidney stone disease was performed for 12,123 individuals with kidney stone disease and 417,378 controls from the UK Biobank and BioBank Japan. a is a quantile-quantile plot of observed vs. expected p-values. The $\lambda_{\mathrm{GC}}$ demonstrated some inflation (1.0957), but the LD score regression (LDSC) intercept of 0.9997, with an attenuation ratio of 0.0075 indicated that the inflation was largely due to polygenicity and the large sample size. $\mathbf{b}$ is a Manhattan plot showing the genome-wide $p$ values $(-\log 10)$ plotted against their respective positions on each of the autosomes. The horizontal red line shows the genome-wide significance threshold of $5.0 \times 10^{-8}$. Loci have been labeled with the primary candidate gene at each locus, as shown in Table 1. Previously unreported GWAS-discovered kidney stone loci are highlighted in red

(Table 2). No correlation was found between rs17216707 genotype and serum phosphate, $\mathrm{PTH}, 25$-hydroxyvitamin $\mathrm{D}$ concentration or urinary calcium excretion. Intake of medications including steroids and diuretics was comparable across genotypes (Supplementary Fig. 4A).

Calcium-sensing receptor signaling pathway associated loci. Five of the identified loci are linked to genes that are predicted to influence CaSR signaling (DGKD, DGKH, WDR72, GIPC1, and $B C R$ ) (Fig. 2). rs13003198 is $\sim 6 \mathrm{~kb}$ upstream of $D G K D$, encoding diacylglycerol kinase delta (DGKD); rs1037271 is an intronic variant in $D G K H$, encoding diacylglycerol kinase eta (DGKH). DGKD and DGKH phosphorylate diacylglcerol, a component of the intracellular CaSR-signaling pathway inducing CaSR-mediated membrane ruffling and activating protein kinase $\mathrm{C}$ signaling cascades including MAPK and intracellular calcium release ${ }^{23,24}$ (Fig. 2). rs578595 is an intronic variant in WDR72 encoding WD repeat domain 72 (WDR72) and rs3760702 is $~ 300$ bp upstream of GIPC1 that encodes Regulator of G-protein signaling 19 Interacting Protein 1 (GIPC1). Both WDR72 and GIPC1 are thought to play a role in clathrin-mediated endocytosis, a process central to sustained intracellular CaSR signaling ${ }^{24-27}$ (Fig. 2). rs13054904 is $\sim 110 \mathrm{~kb}$ upstream of $B C R$, encoding a RACl (Rac Family Small GTPase 1) GTPase-activating protein known as Breakpoint Cluster Region (BCR) ${ }^{28}$. RAC1 activation is induced by CaSR ligand binding and mediates CaSR-induced membrane ruffling $^{23}$ (Fig. 2). Of the 20 genome-wide significant loci discovered in the meta-analysis of British and Japanese populations, this was the only locus that did not reach nominal significance in one of the two populations (British: $p=3.30 \times 10^{-12}$; Japanese: $p=0.505$ ), suggesting that this locus predisposes to renal stones in European but not East Asian populations. In addition, a previously reported association between the CaSR-associated intronic SNP rs7627468 and nephrolithiasis was confirmed ( $p=$ $\left.3.5 \times 10^{-5}\right)^{9}$.

Gain-of-function mutations in components of the CaSRsignaling pathway result in autosomal dominant hypocalcaemia (ADH, OMIM 601198, 615361), which is associated with hypercalciuria in $\sim 10 \%$ of individuals ${ }^{29,30}$. $\mathrm{ADH}$-associated mutations result in a gain-of-function in CaSR-intracellular signaling in vitro via pathways including intracellular calcium ions and MAPK $29,31,32$. We hypothesized that the nephrolithiasis- associated loci linked to CaSR-signaling associate with enhanced CaSR signal transduction resulting in a biochemical phenotype mimicking an attenuated form of ADH. DGKD and DGKH were selected for further analysis based on their potential to influence both membrane ruffling and protein kinase C CaSR-signaling pathways (Fig. 2).

The DGKD increased-risk allele rs838717 (G) (top DGKDassociated SNP in the UK Biobank GWAS, Supplementary Table 3, linkage disequilibrium with $\mathrm{rs} 13003198 \mathrm{r}^{2}=0.53$ ) associated with increased 24 -h urinary calcium excretion in male stone formers (mean 24-h urinary calcium excretion $7.27 \mathrm{mmol}$ (GG) vs. $4.54 \mathrm{mmol}(\mathrm{AA})$; trend test $p$-value for additive effect $=$ 0.017) (Table 2), consistent with enhanced CaSR-signal transduction. No association of the DGKD increased-risk allele rs838717 (G) with urinary calcium excretion was identified in female stone formers. No correlations were observed between genotype and serum calcium, phosphate, PTH, 25-hydroxyvitamin D concentrations or number of stone episodes.

The DGKH increased-risk allele rs1170174 (A) (top DGKHassociated SNP in the UK Biobank GWAS, Supplementary Table 3, linkage disequilibrium with $\mathrm{rs} 13003198 \mathrm{r}^{2}=0.3$ ) did not associate with biochemical phenotype or stone recurrence. However, the sample size of homozygotes was small (AA, $n=6$ ) and prior to Bonferroni correction, a suggestive association was detected with urinary calcium excretion in male stone formers (mean 24-h urinary calcium excretion $8.14 \mathrm{mmol}$ (AA) vs. 5.09 mmol (GG), student's $t$ test $p=0.0503$ ) (Supplementary Table 7). Prescription of medications, including steroids and diuretics, was comparable across DGKD and DGKH genotypes (Supplementary Fig. 4B, 4C).

To investigate the role of DGKD in CaSR-signaling via the MAPK pathway, HEK-CaSR-SRE and HEK-CaSR cells were treated with scrambled or DGKD targeted siRNA and intracellular MAPK responses to alterations in extracellular calcium concentration assessed via SRE and ERK-phosphorylation (pERK) assays, respectively. Treatment with $D G K D$ targeted siRNA resulted in a reduction in DGKD expression when compared to cells treated with scrambled siRNA without alteration in CaSR expression (Fig. 3a-c and Supplementary Fig. 5A, B). SRE and pERK responses were significantly decreased in cells with reduced DGKD expression (DGKD-KD) when compared to cells with baseline DGKD expression (WT) (SRE maximal response DGKD$\mathrm{KD}=5.28$ fold change, $95 \%$ confidence interval $(\mathrm{CI})=4.77-5.79$ 


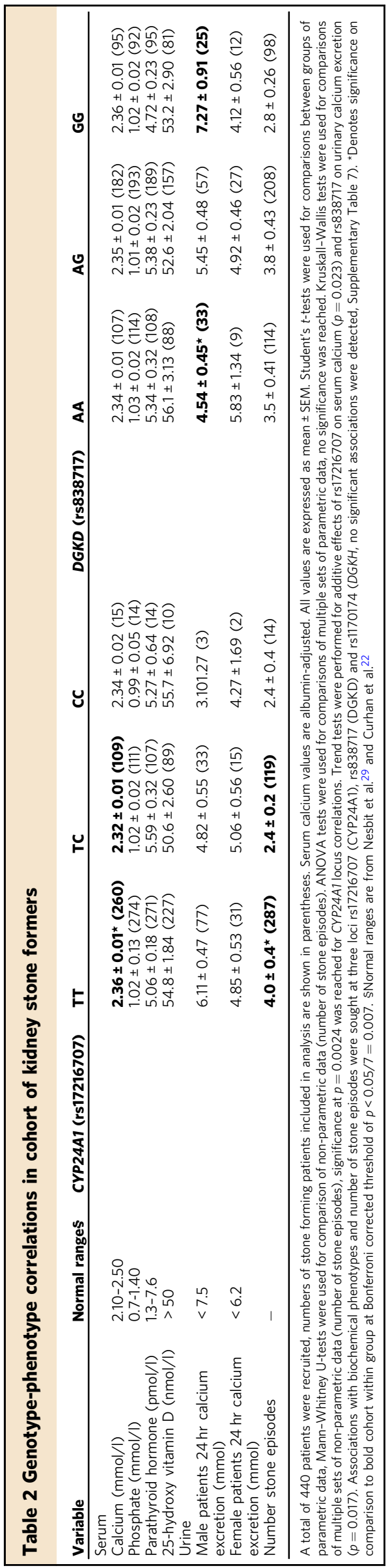

vs. $\mathrm{WT}=7.20$ fold change, $95 \% \mathrm{CI}=6.46-7.93, \mathrm{~F}$ test $p=0.0065$; pERK maximal response $\mathrm{DGKD}-\mathrm{KD}=24.77, \quad 95 \% \mathrm{CI}=$ $22.16-27.38$ vs. $\mathrm{WT}=39.46$ fold change, $95 \% \mathrm{CI}=34.07-44.84, \mathrm{~F}$ test $p=0.0056)$. Cinacalcet rectified this loss-of-function in SREreporter assays (DGKD-KD $+5 \mathrm{nM}$ cinacalcet, maximal response $=7.62$ fold change, 95\% CI =5.98-9.27) (Fig. 3d-e and Supplementary Fig. 5C).

Furthermore, to investigate the role of DGKD in CaSRsignaling via intracellular calcium mobilization, HEK-CaSRNFAT and HEK-CaSR cells were treated with scrambled or DGKD targeted siRNA and intracellular NFAT and intracellular calcium responses to alterations in extracellular calcium concentration assessed via NFAT-reporter and Fluo-4 calcium assays, respectively. Intracellular calcium responses were unaffected by a reduction in DGKD expression (NFAT maximal response DGKD$\mathrm{KD}=2.91$ fold change, $95 \% \mathrm{CI}=2.69-3.12 v s . \mathrm{WT}=2.85$ fold change, $95 \% \mathrm{CI}=2.62-3.08, \mathrm{~F}$ test $p=0.73$; Fluo-4 maximal response $\mathrm{DGKD}-\mathrm{KD}=96.16,95 \% \mathrm{CI}=89.83-102.5$ vs. $\mathrm{WT}=$ 96.78 fold change, 95\% CI=92.76-100.8, F test $p=0.83$ ) (Supplementary Fig. 5D, E).

\section{Discussion}

Our study, which represents the largest kidney stone GWAS to date and integrates data from 12,123 stone formers and 417,378 controls from British and Japanese ancestries, identifies 20 genetic loci associated with nephrolithiasis, seven of which have not previously been reported to associate with kidney stone disease at GWAS. The genes implicated by our GWAS are disproportionately expressed in the renal cortex, with enrichment for biological pathways and gene ontologies involving solute transport, renal physiology and calcium homeostasis.

Our findings highlight the role of vitamin $\mathrm{D}$ catabolism in kidney stone formation. Thus, we identify a locus $\sim 38 \mathrm{~kb}$ upstream of CYP24A1 (rs17216707) and demonstrate that genotype at this locus is associated with serum calcium concentration and stone recurrence episodes in a cohort of kidney stone patients. These findings support our hypothesis that the rs17216707 increasedrisk allele is associated with a relative hypercalcaemia and reduced activity of the 24-hydroxylase enzyme. Patients with loss-offunction CYP24A1 mutations have been successfully treated with inhibitors of vitamin D synthesis including fluconazole, similar therapies may be useful in rs17216707 (TT) recurrent kidney stone formers ${ }^{33}$. Furthermore, vitamin D supplementation in patients with biallelic loss-of-function mutations in CYP24A1 cause nephrocalcinosis ${ }^{21}$. We predict that rs17216707 (TT) individuals may similarly display an increased sensitivity to vitamin D. The National Institute of Clinical Excellence (NICE) recommends that all adults living in the UK should take daily vitamin D supplementation. However, our findings suggest that supplementation may put individuals homozygous for the CYP24A1 increasedrisk allele at risk of kidney stones; studies are required to investigate this hypothesis further.

Five of the loci identified at GWAS are linked to genes that are predicted to influence CaSR signaling: DGKD, DGKH, WDR72, GIPC1, and BCR. The CaSR is a G-protein coupled receptor that is highly expressed in the parathyroid and kidneys and has a central role in calcium homeostasis, increasing renal calcium reabsorption and stimulating PTH release to enhance bone resorption, urinary calcium reabsorption, and renal synthesis of 1,25-dihydroxyvitamin $\mathrm{D}^{34}$.

The DGKD increased-risk allele associates with urinary calcium excretion in male stone formers but not in female stone formers. This is probably due to a lack of power as a result of the small sample size of female stone formers, however it is interesting to note that heritability of stone disease is lower in women than in men ${ }^{5}$. 


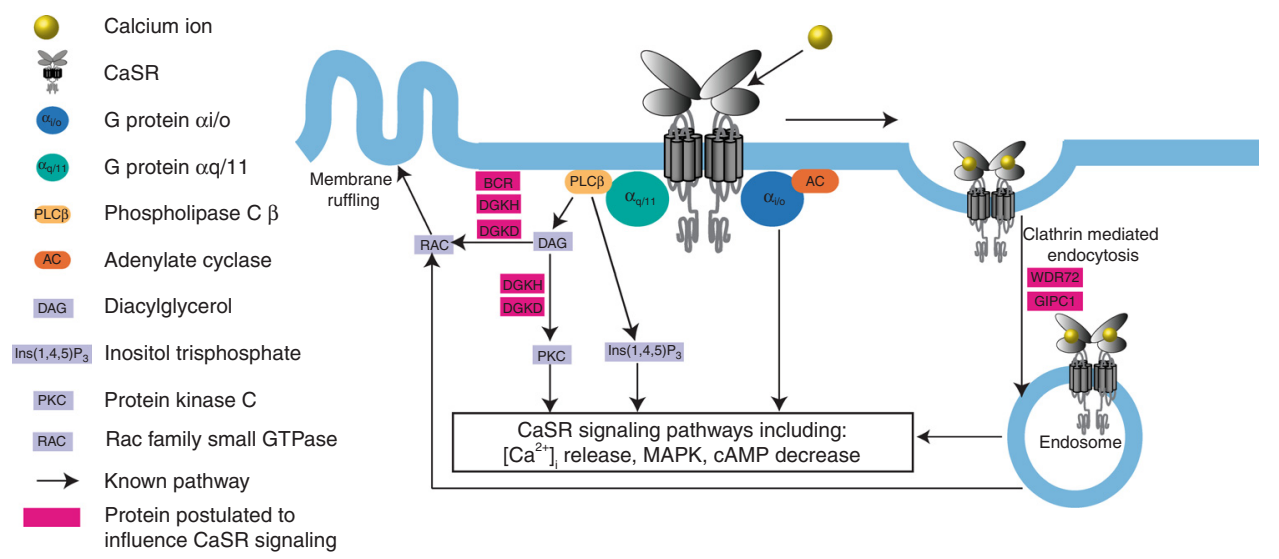

Fig. 2 Schematic model for CaSR signaling. Ligand binding of calcium ions (yellow) by the G protein coupled receptor CaSR (gray) results in G proteindependent stimulation via $\mathrm{G} \alpha_{\mathrm{q} / 11}$ (turquoise) or $\mathrm{G}_{\mathrm{i} / \mathrm{o}}$ (blue) causing stimulation of intracellular signaling pathways including intracellular calcium ([Ca $\left.{ }^{2+}\right]_{\mathrm{i}}$ ) release, MAPK stimulation or CAMP reduction. $G \alpha_{q / 11}$ signals via inositol 1,4,5-trisphosphate (IP3) and diacylglycerol (DAG). DAG leads to protein kinase $\mathrm{C}$ (PKC) stimulation along with RAC activation, which results in membrane ruffling. Following calcium ion binding the CaSR is internalized via clathrinmediated endocytosis where signaling continues via the endosome. Proteins postulated to influence CaSR-signaling and their potential sites of action are shown in magenta

Furthermore, no correlations were identified with serum calcium concentrations despite this being the prominent phenotype in $\mathrm{ADH}$ patients. Reduced expression of DGKD results in decreased CaSRmediated intracellular signaling via the MAPK pathway in vitro, but not via intracellular calcium mobilization. These findings provide evidence that DGKD influences CaSR-mediated signal transduction and suggest that the $D G K D$ increased-risk allele may associate with a relative increase in DGKD expression thereby enhancing CaSRmediated signaling via the MAPK pathway whilst leaving signaling via the intracellular calcium pathway unaffected. This biased signaling may provide an explanation for the observed correlation of the DGKD increased-risk allele rs $838717(\mathrm{G})$ with increased urinary calcium excretion but not serum calcium concentration (Table 2). Calcilytics, including NPS-2143 and ronacaleret, rectify enhanced CaSR-mediated signaling in vitro and biochemical phenotypes in mouse models of $\mathrm{ADH}^{32,35,36}$. We predict that the development of biased calcilytics may provide a novel, targeted therapeutic approach to reduce urinary calcium excretion in recurrent stone formers carrying CaSR-associated increased-risk alleles.

In conclusion, this study identifies 20 loci linked to kidney stone formation, seven of which are have not previously been reported to associate with kidney stone disease, and reveals the importance of vitamin D metabolism pathways and enhanced CaSR-signaling in the pathogenesis of nephrolithiasis. Our findings suggest that there may be a role for genetic testing to identify individuals in whom vitamin D supplementation should be used with caution and to facilitate a precision-medicine approach for the treatment of recurrent kidney stone disease, whereby targeting of the CaSR-signaling or vitamin D metabolism pathways may be beneficial in the treatment of a subset of patients with nephrolithiasis; further studies are required.

\footnotetext{
Methods

Study participants. The UK Biobank resource was utilized for the UK-based GWAS. The UK Biobank is a prospective cohort study of $\sim 500,000$ individuals from the UK, aged between 40 and 69, who have had whole-genome genotyping undertaken, and have allowed linkage of these data with their medical records 12,37 . ICD-10 and OPCS codes and a single UK Biobank self-reported operation code for kidney stone surgery were used to identify individuals with a history of nephrolithiasis (Supplementary Table 1). Individuals were excluded if they were recorded to have a disorder of calcium homeostasis, malabsorption, or other condition known to predispose to kidney stone disease (Supplementary Table 2). Following quality control (QC) 6,536 UK Biobank participants were identified as cases and
} 388,508 individuals as controls (Supplementary Table 8).
In Japanese patients a diagnosis of nephrolithiasis was confirmed by enrolling physicians; patients with bladder stones were excluded. Information regarding conditions known to predispose to kidney stones was unavailable. DNA samples of 5,587 nephrolithiasis patients were obtained from BioBank Japan ${ }^{13}$. Controls (28,870 individuals) were identified from four population-based cohorts, including the JPHC (Japan Public Health Center)-based prospective study ${ }^{38}$, the J-MICC (Japan Multi-Institutional Collaborative Cohort) study ${ }^{39}$, IMM (Iwate Tohoku Medical Megabank Organization) and ToMMo (Tohoku Medical Megabank Organization) ${ }^{40}$, (Supplementary Table 9).

Four hundred and forty patients attending the Oxford University Hospitals NHS Foundation Trust for treatment of kidney stones were enrolled into the Oxford University Hospitals NHS Foundation Trust Biobank of Kidney Stone Formers, following informed consent. These patients made up the validation cohort. Clinical data including urological history, past medical history, medication intake, and family history was recorded along with details of serum and urinary biochemistry. Whole blood and urine samples were stored at $-80^{\circ} \mathrm{C}$.

Ethical approval. UK Biobank has approval from the North West Multi-Centre Research Ethics Committee (11/NW/0382), and this study (Epidemiology of Kidney Stone Disease) has UK Biobank study ID 885. Ethical committees at the Institute of Medical Science, The University of Tokyo and RIKEN approved the project (study IDs 29-74-A0215 and 17-17-16(3)). Collection of clinical data and biological samples from kidney stone patients attending the Oxford University Hospitals NHS Foundation Trust was approved by the University of Oxford under the Oxford Radcliffe Biobank research tissue bank ethics $(09 / \mathrm{H} 0606 / 5+5)$. All patients provided written informed consent.

Genotyping. The UK Biobank contains genotypes of 488,377 participants who were genotyped on two very similar genotyping arrays: UK BiLEVE Axiom Array (807,411 markers; 49,950 participants), and UK Biobank Axiom Array (825,927 markers; 438,427 participants). The two arrays are very similar, sharing $~ 95 \%$ of marker content. Genotypes were called from the array intensity data, in 106 batches of approximately 4700 samples each using a custom genotype-calling pipeline ${ }^{41}$.

Japanese samples were genotyped with Illumina HumanOmniExpressExome BeadChip or a combination of the Illumina HumanOmniExpress and HumanExome BeadChips ${ }^{42,43}$.

For genotyping of the validation cohort, DNA was extracted from whole blood samples using the Maxwell 16 Tissue DNA purification kit (Promega). SNP genotyping was undertaken using TaqMan SNP Genotyping Assays (ThermoFisher, Supplementary Table 11) and Type-it ${ }^{\circledR}$ Fast SNP Probe PCR Kit (Qiagen).

Quality Control. Quality Control (QC) of the UK Biobank data was performed using PLINK ${ }^{44}$ v1.9 and R v3.3.1. All SNPs with a call rate $<90 \%$ were removed, accounting for the two different genotyping platforms used to genotype the individuals. Sample-level QC was undertaken and individuals excluded with one or more of the following: (1) call rate $<98 \%$, (2) discrepancy between genetically inferred sex (Data Field 22001) and self-reported sex (Data Field 31), or individuals with sex chromosome aneuploidy (Data Field 22019), (3) heterozygosity > 
a

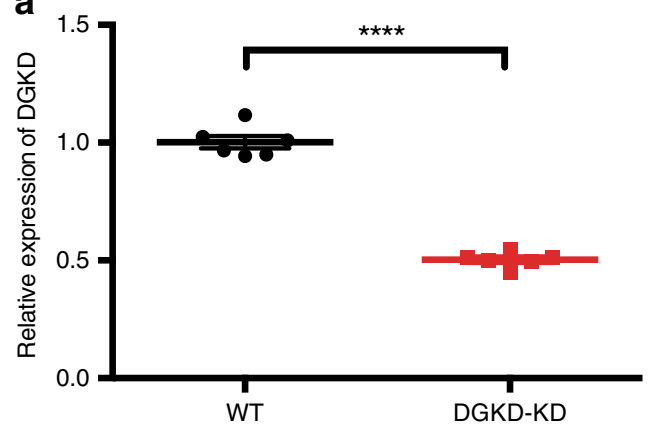

C

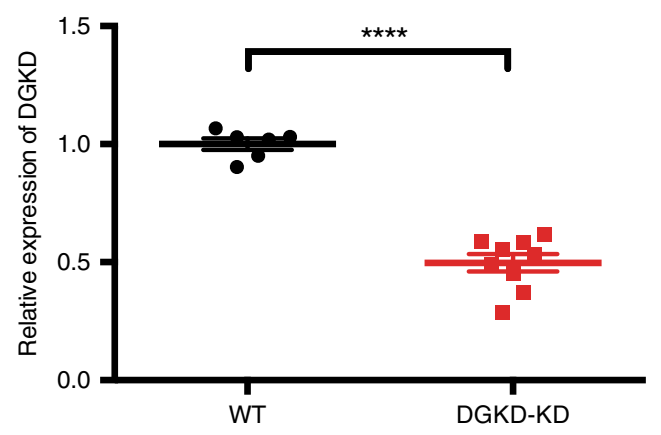

e

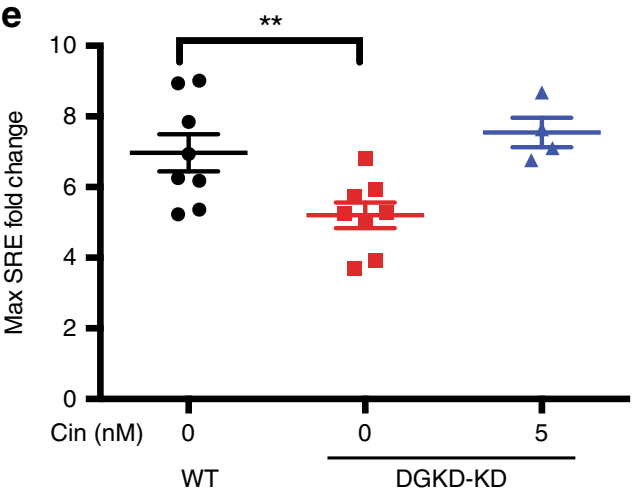

b

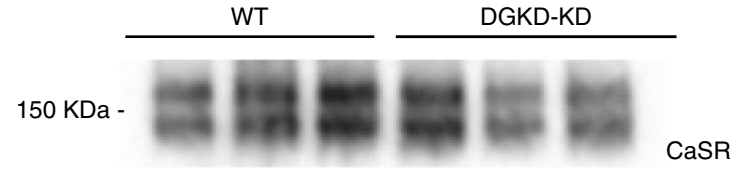

$75 \mathrm{KDa}$ -

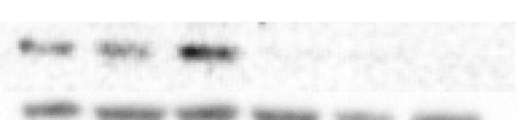

$50 \mathrm{KDa}$ aSR DGKD $\alpha$-Tubulin d

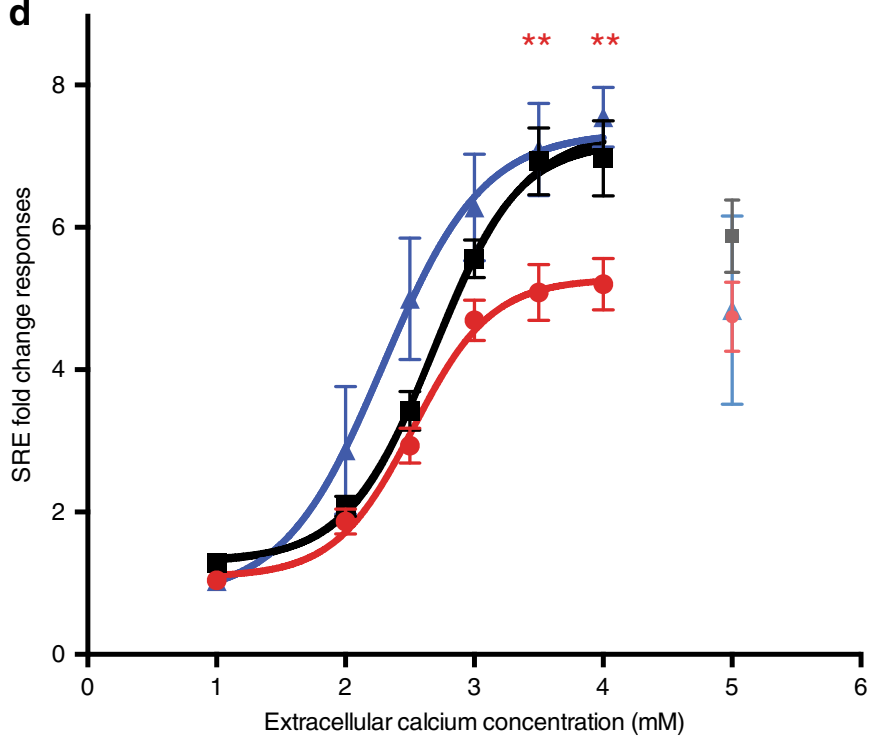

WT Max. response $=7.20(95 \% \mathrm{Cl}=6.46-7.93)$

DGKD-KD Max. response $=5.28(95 \% \mathrm{Cl}=4.77-5.79)^{\star \star}$

А DGKD-KD+Cin Max response $=7.62(95 \% \mathrm{Cl}=5.98-9.27)$

Fig. 3 CaSR-mediated SRE responses following DGKD knockdown and effect of cinacalcet treatment in HEK-CaSR-SRE cells. a Relative expression of DGKD, as assessed by quantitative real-time PCR of HEK-CaSR-SRE cells treated with scrambled (WT) or DGKD (DGKD-KD) siRNA and used for SRE experiments. Samples were normalized to a geometric mean of four housekeeper genes: PGK1, GAPDH, TUB1A, CDNK1B. $n=8$ biologically independent transfections. b Representative western blot of lysates from HEK-CaSR cells treated with scrambled or DGKD siRNA and used for SRE experiments. $\alpha-$ Tubulin was used as a loading control. c Relative expression of DGKD, as assessed by densitometry of western blots from cells treated with scrambled or DGKD siRNA demonstrating a $50 \%$ reduction in expression of DGKD following treatment with DGKD siRNA. Samples were normalized to PGK1. $n=6$ biologically independent transfections for WT, $n=9$ biologically independent transfections for DGKD-KD. d SRE responses of HEK-CaSR-SRE cells in response to changes in extracellular calcium concentration. Cells were treated with scrambled (WT) or DGKD (DGKD-KD) siRNA. The responses \pm SEM are shown for $\mathrm{n}=8$ biologically independent transfections for WT and DGKD-KD cells and $n=4$ biologically independent transfections for DGKD-KD $+5 \mathrm{nM}$ cinacalcet cells. Treatment with DGKD siRNA led to a reduction in maximal response (red line) compared to cells treated with scrambled siRNA (black line). This lossof-function could be rectified by treatment with $5 \mathrm{nM}$ cinacalcet (blue line). Post desensitization points are shown but were not included in the analysis (gray, light red, and light blue). e Mean maximal responses with SEM of cells treated with scrambled siRNA (WT, black), DGKD siRNA (DGKD-KD, red) and DGKD siRNA incubated with $5 \mathrm{nM}$ cinacalcet (blue). Statistical comparisons of maximal response were undertaken using $\mathrm{F}$ test. Student's t-tests were used to compare relative expression. Two-way ANOVA was used to compare points on dose response curve with reference to WT. Data are shown as mean \pm SEM with ${ }^{\star \star} p<0.01,{ }^{\star \star \star \star} p<0.0001$. Source data are provided as a Source Data file

3 standard deviations from the mean (calculated using UK Biobank's PCA-adjusted
heterozygosity values, Data Field 20004). Individuals were then excluded who were
not flagged by UK Biobank as having white British ancestry (on the basis of
principal component analysis and self-reporting as "British" - Data Field 22006).
Data was merged with publicly available data from the 1000 Genomes Project ${ }^{45}$
and principal component analysis (PCA) performed using flashpca ${ }^{46}$ to confirm
that the white British ancestry individuals from UK Biobank overlapped with the
"GBR" individuals from the 1000 Genomes Project. BOLT-LMM was used in
analysis and therefore there were no sample exclusions based on relatedness ${ }^{14}$. In total, 86,693 individuals were excluded based on the above criteria. SNP-level QC was performed by excluding SNPs with Hardy-Weinberg equilibrium (HWE)

$p<10^{-4},<98 \%$ call rate, and minor allele frequency (MAF) $<1 \%$. Overall, 237,245 SNPs were excluded in total. Finally, six individuals were excluded who harboured an abnormal number of SNPs with a minor allele count of 1 , or were visual outliers when autosomal heterozygosity was plotted against call rate. This resulted in a final dataset of 401,667 individuals and 547,011 SNPs. Following QC, individuals were excluded who were recorded to have a disorder of calcium homeostasis, malabsorption, or other condition known to predispose to kidney stone disease 
(Supplementary Table 2). Subsequent case ascertainment was performed using the list of ICD-10 and OPCS codes for kidney and ureteric stones (Supplementary Table 1).

In the Japanese dataset samples were excluded if (i) call rate was $<0.98$, (ii) they were from closely related individuals identified by identity-by-descent analysis, (iii) the samples were sex-mismatched with a lack of information, or (iv) they were non-East Asian outliers identified by principal component analysis of the studied samples and the three major reference populations (Africans, Europeans, and East Asians) in the International HapMap Project ${ }^{47}$. Standard quality-control criteria for variants were applied, excluding those with (i) SNP call rate $<0.99$, (ii) minor allele frequency $<1 \%$, and (iii) Hardy-Weinberg equilibrium $P$ value $<1.0 \times 10^{-6}$.

Imputation. UK Biobank phasing on the autosomes was performed using SHAPEIT3 ${ }^{48}$, using the 1000 Genomes Phase 3 dataset as a reference panel. For imputation, both the HRC (Haplotype Reference Consortium) reference panel ${ }^{49}$ and a merged UK10K/1000 Genomes Phase 3 panel were used. This resulted in a dataset with 92,693,895 autosomal SNPs, short indels and large structural variants. Imputation files were released in the BGEN (v1.2) file format ${ }^{41}$.

In the case of Japanese data genotypes were prephased with $\mathrm{MACH}^{50}$ and dosages imputed with minimac and the 1000 Genomes Project Phase 1 (version 3) East Asian reference haplotypes ${ }^{45}$.

Association analyses. In the UK Biobank dataset genome-wide association analysis was undertaken across 547,011 genotyped SNPs and $\sim 8.4$ million imputed SNPs with MAF $\geq 0.01$ and Info Score $\geq 0.9$, using a linear mixed non-infinitesimal model implemented in BOLT-LMM v2.351. A reference genetic map file for hg19 and a reference linkage disequilibrium (LD) score file for European-ancestry individuals included in the BOLT-LMM package in the analysis was used. Two covariates were used in the association study: genetic sex, and the genotyping platform (to account for array effects). Quantile-quantile and Manhattan plots were generated using FUMA.

The genomic inflation factor $\left(\lambda_{\mathrm{GC}}\right)$ and a value for the $\lambda_{\mathrm{GC}}$ adjusted to a sample size of $1000\left(\lambda_{1000}\right)$ are given for the UK Biobank GWAS, the BioBank Japan GWAS $^{11}$, and the trans-ethnic meta-analysis (Supplementary Table 10).

The LD score regression intercept $\mathrm{t}^{45}$ of 0.9997 with an attenuation ratio of 0.0075 indicated minimal inflation when adjusted for the large sample size. Conditional analysis at each associated locus was performed by conditioning on the allelic dosage (calculated using QCTOOL v2) of the most significantly associated SNP at each locus.

In the Japanese dataset GWAS was conducted using a logistic regression model by incorporating age, sex, and the top 10 principal components as covariates. Control individuals were younger and more commonly male; thus, these factors were included as covariates during association analysis.

Trans-ethnic meta-analysis was performed using the summary statistics from the UK and Japanese GWAS datasets (12,123 cases and 417,378 controls). An imputation quality score (RSQR) threshold of $>0.5$ was applied to the Japanese GWAS SNPs $s^{52}$ prior to performing a fixed-effects meta-analysis using GWAMA ${ }^{53}$, using $\sim 5$ million SNPs common to both GWAS datasets. Quantile-quantile and Manhattan plots were generated using FUMA ${ }^{19}$.

In silico analyses. The summary statistics from the GWAS meta-analysis were analyzed in FUMA ${ }^{19}$ v1.3.3c, selecting UK Biobank Release 2 (White British) as the population reference panel (as the vast majority of individuals in the meta-analysis are of British rather than Japanese ethnicity). Functionally annotated SNPs were mapped to genes based on genomic position and annotations obtained from ANNOVAR, using positional mapping in FUMA (Supplementary Table 5) ${ }^{54}$. MAGMA (implemented in FUMA) was used to perform a gene-property analysis in order to identify particular tissue types relevant to kidney stones. This analysis determines if tissue-specific differential expression levels are predictive of the association of a gene with kidney stones, across 53 different tissues taken from the GTEx v7 database ${ }^{55}$ (Supplementary Fig. 2).

In order to gain insight into the biological pathways implicated by the FUMAprioritized genes, a gene set analysis was implemented using the GENE2FUNC tool in FUMA using the 54 positionally mapped genes with unique Entrez IDs and gene symbols. The following parameters were applied: Benjamini-Hochberg false discovery rate (FDR) for multiple testing correction, adjusted $p$-value cut-off $=$ 0.0025 , minimum number of overlapped genes $=2$, GTEx v7 RNA-Seq expression data. Hypergeometric tests were performed to test if genes of interest are overrepresented in any of the pre-defined gene sets in GO biological processes (MsigDB v6.1) (Supplementary Fig. 3).

Genotype-phenotype correlations. Associations were sought between genotype and serum calcium (albumin adjusted), phosphate, parathyroid hormone, and 25hydroxyvitamin $\mathrm{D}$, and urinary calcium excretion and number of stone episodes. Patients were excluded from inclusion in genotype-phenotype correlations if they were known to have a disorder of calcium homeostasis, malabsorption, or other condition known to predispose to kidney stone disease.
Characterization of the calcium-sensing receptor pathway. Functional studies were undertaken using HEK293 cells (ATCC ${ }^{\circ}$ CRL-1573 $3^{\mathrm{m}}$ ) that had been transfected to stably express calcium-sensing receptors (CaSRs) (HEK-CaSR cells). In addition, cells used for serum-response element (SRE) and nuclear factor or activated $T$-cells (NFAT) assays were stably transfected to express luciferase under the control of SRE (HEK-CaSR-SRE cells) or NFAT (HEK-CaSR-NFAT cells), respectively. Cells were transfected with $10 \mathrm{nM}$ scrambled or DGKD siRNA (Qiagen) using lipofectamine RNAiMAX (Thermo Fisher Scientific) $72 \mathrm{~h}$ before experiments and maintained in DMEM-Glutamax media (Thermo Fisher Scientific) with $10 \%$ FBS (Gibco) and $400 \mu \mathrm{g} / \mathrm{ml}$ geneticin (Thermo Fisher Scientific) and $200 \mu \mathrm{g} / \mathrm{ml}$ hygromycin (Invitrogen) at $37^{\circ} \mathrm{C}, 5 \% \mathrm{CO}_{2}$.

Successful knockdown of DGKD and maintenance of CaSR expression was confirmed via quantitative reverse transcriptase PCR (qRT-PCR) and western blot analyses. qRT-PCR analyses were performed in quadruplicate using Power SYBR Green Cells-to-CT ${ }^{\text {tw }}$ Kit (Life Technologies), DGKD, CASR, PGK1, GAPDH, $T U B 1 A, C D N K 1 B$ specific primers (Qiagen, Supplementary Table 11), and a RotorGene Q real-time cycler (Qiagen Inc, Valencia, CA). Samples were normalized to a geometric mean of four housekeeper genes: PGK1, GAPDH, TUB1A, CDNK1B. Western blot analyses were undertaken using anti-DGKD (SAB1300472; Sigma; 1:1000), anti-CaSR (5C10, ADD; ab19347; Abcam; 1:1000), and anti- $\alpha-$ Tubulin (T5168; Sigma; 1:2000). The western blots were visualized using an Immuno-Star Western C kit (Bio-Rad) on a Bio-Rad Chemidoc XRS + system and relative expression of DGKD and CaSR were quantified by denistometry using ImageJ software. Both the $140 \mathrm{kDa}$ immature CaSR band and the $160 \mathrm{kDa}$ glycosylated band were considered in densitometry calculations. Captured images are included in the Source Data File.

To perform SRE response assays, at $60 \mathrm{~h}$ post transfection HEK-CaSR-SRE cells were incubated in $0.05 \%$ fetal bovine serum media with $0.45 \mathrm{mM}$ calcium for $12 \mathrm{~h}$, reducing extracellular calcium concentration and thus inducing basal cellular CaSR-mediated responses whilst maintaining cellular viability. At $72 \mathrm{~h}$ the media was changed to varying concentrations of extracellular calcium $(0.1-5 \mathrm{mM})$, with either $5 \mathrm{nM}$ cinacalcet or equivalent volume of DMSO (final concentration of DMSO $0.0001 \%$ ), and the cells were incubated for a further $4 \mathrm{~h}$ at $37^{\circ} \mathrm{C}$. Cinacalcet (AMG-073 HCL) was obtained from Cambridge Bioscience (catalog CAY16042) and dissolved in DMSO prior to use in in vitro studies. Cells were lysed and luciferase activity measured using Luciferase Assay System (Promega) on a PHERAstar microplate reader (BMG Labtech). Assays were performed in $>4$ biological replicates (independently transfected wells, performed on at least 4 different days). Nonlinear regression of concentration-response curves was performed with GraphPad Prism for determinations of maximal response.

To perform ERK phosphorylation assays HEK-CaSR cells were seeded in 96well plates at $60 \mathrm{~h}$ post transfection. Cells were fasted for $4 \mathrm{~h}$ in $0.1 \mathrm{mM}$ extracellular calcium then stimulated for 4 min with varying concentrations of extracellular calcium (0.1-5 mM), and lysed in Alphascreen Surefire lysis buffer. Alphascreen Surefire ERK phosphorylation assays were performed on whole cell lysates, as reported, and the fluorescence signal measured using a PHERAStar microplate reader (BMG Labtech $)^{56}$. Assays were performed in $>4$ biological replicates (independently transfected wells, performed on at least 4 different days). Nonlinear regression of concentration-response curves was performed with GraphPad Prism for determinations of maximal response.

To perform NFAT response assays, at $60 \mathrm{~h}$ post transfection HEK-CaSR-NFAT cells were incubated in $0.05 \%$ fetal bovine serum media with $0.45 \mathrm{mM}$ calcium for $12 \mathrm{~h}$, reducing extracellular calcium concentration and thus inducing basal cellular CaSR-mediated responses whilst maintaining cellular viability. At $72 \mathrm{~h}$ the media was changed to varying concentrations of extracellular calcium $(0.1-5 \mathrm{mM})$ and the cells were incubated for a further $4 \mathrm{~h}$ at $37^{\circ} \mathrm{C}$. Cells were lysed and luciferase activity measured using Luciferase Assay System (Promega) on a PHERAstar microplate reader (BMG Labtech). Assays were performed in $>4$ biological replicates (independently transfected wells, performed on at least 4 different days). Nonlinear regression of concentration-response curves was performed with GraphPad Prism for determinations of maximal response.

To measure intracellular calcium responses, at $60 \mathrm{~h}$ post transfection HEKCaSR cells were plated in 96-well plates. At $70 \mathrm{~h}$ post transfection cells were washed with $100 \mu$ l Complete Imaging Buffer $(150 \mathrm{mM} \mathrm{NaCl}, 2.6 \mathrm{mM} \mathrm{KCl}, 1.18 \mathrm{mM}$ $\mathrm{MgCl}_{2}, 10 \mathrm{mM}$ HEPES, $0.1 \mathrm{mM} \mathrm{CaCl} 2, \mathrm{pH} 7.4$ ), loaded with Fluo- 4 dye (Complete Imaging Buffer supplemented with; $1 \mu \mathrm{M}$ Fluo- $4 \mathrm{AM}, 0.01 \%$ pluronic F-127, 0.5\% BSA), and incubated for $60 \mathrm{~min}$ at $37^{\circ} \mathrm{C}$. Cells were washed again before the addition of a further $100 \mu \mathrm{l}$ of Complete Imaging Buffer and incubated for $30 \mathrm{~min}$ at room temperature in the dark. Using an automated system, calcium chloride was injected into wells to achieve extracellular calcium concentrations ranging from 0.05 to $5 \mathrm{mM}$. Control wells were injected with $10 \mu \mathrm{M}$ ionomycin. Changes in intracellular calcium concentrations were recorded via detection of fluorescence for $30 \mathrm{~s}$ using a PHERAstar microplate reader (BMG Labtech) at $37^{\circ} \mathrm{C}$ with an excitation filter of $485 \mathrm{~nm}$ and an emission filter of $520 \mathrm{~nm}$. The peak mean fluorescence ratio of the transient response following each individual stimulus was measured using MARS data analysis software (BMG Labtech). Relative fluorescence units were normalized to the fluorescence stimulated by ionomycin to account for differences in cell number and loading efficiency and then further normalized to the maximum response observed for the cells treated with scrambled siRNA $^{57,58}$. Assays were performed using 4 biological replicates (independently transfected wells, performed on at least 4 different days). Nonlinear regression of 
concentration-response curves was performed with GraphPad Prism for determinations of maximal response.

Statistics. For genotype-phenotype correlations, 2-tailed Student's $t$-tests were used for parametric data. Mann-Whitney U-tests were used for comparison of non-parametric data (number of stone episodes). ANOVA tests were used for comparisons of multiple sets of parametric data. Kruskall-Wallis tests were used for comparisons of multiple sets of non-parametric data (number of stone episodes). Significance was defined as $p<0.05$ after Bonferroni correction for 7 tests on each set of data, thus $p<0.05 / 7=0.007$. To calculate $p$-values for the additive effects of rs17216707 on serum calcium concentration and rs838717 on urinary calcium excretion, we performed a non-parametric test for trend across ordered groups (an extension of the Wilcoxon rank-sum test) ${ }^{59}$, using the nptrend function in Stata $^{60}$. For in vitro studies statistical comparisons were made with 2-tailed Student's $t$-tests, maximal responses were compared using the $F$-test, and responses at each extracellular calcium concentration were compared using a 2-way ANOVA with Tukey's multiple-comparisons test using GraphPad Prism 6 29,58 .

Reporting Summary. Further information on research design is available in the Nature Research Reporting Summary linked to this article.

\section{Data availability}

Full UK Biobank data are available by direct application to UK Biobank. Full GWAS summary statistics from the UK Biobank GWAS and the UK-Japanese meta-analysis can be found at: https://doi.org/10.5287/bodleian:2NEEgv2QD. Source data for Fig. 3, Table 2, and Supplementary Fig. 5 are included in the Source Data file. All other relevant data is available from the authors on request.

Received: 28 January 2019; Accepted: 16 October 2019; Published online: 15 November 2019

\section{References}

1. Scales, C. D., Smith, A. C., Hanley, J. M. \& Saigal, C. S. Urologic Diseases in America Project. Prevalence of kidney stones in the United States. Eur. Urol. 62, 160-165 (2012).

2. Antonelli, J. A., Maalouf, N. M., Pearle, M. S. \& Lotan, Y. Use of the National Health and Nutrition Examination Survey to calculate the impact of obesity and diabetes on cost and prevalence of urolithiasis in 2030. Eur. Urol. 66, 724-729 (2014).

3. Pearle, M. S. et al. Medical management of kidney stones: AUA guideline. J. Urol. 192, 316-324 (2014).

4. Gambaro, G. et al. The risk of chronic kidney disease associated with urolithiasis and its urological treatments: a review. J. Urol. 198, 268-273 (2017).

5. Goldfarb, D. S., Avery, A. R., Beara-Lasic, L., Duncan, G. E. \& Goldberg, J. A twin study of genetic influences on nephrolithiasis in women and men. Kidney Int. Rep. 4, 535-540 (2019).

6. Hunter, D. J. et al. Genetic contribution to renal function and electrolyte balance: a twin study. Clin. Sci. 103, 259-265 (2002).

7. Hemminki, K. et al. Familial risks in urolithiasis in the population of Sweden. BJU Int. 121, 479-485 (2018).

8. Thorleifsson, G. et al. Sequence variants in the CLDN14 gene associate with kidney stones and bone mineral density. Nat. Genet. 41, 926-930 (2009).

9. Oddsson, A. et al. Common and rare variants associated with kidney stones and biochemical traits. Nat. Commun. 6, 7975 (2015).

10. Urabe, Y. et al. A Genome-Wide Association Study of Nephrolithiasis in the Japanese Population Identifies Novel Susceptible Loci at 5q35.3, 7p14.3, and 13q14.1. PLoS Genet. 8, e1002541-e1002547 (2012).

11. Tanikawa, C. et al. Novel risk loci identified in a genome-wide association study of urolithiasis in a Japanese Population. J. Am. Soc. Nephrol. 30, 855-864 (2019).

12. Collins, R. What makes UK Biobank special? Lancet 379, 1173-1174 (2012).

13. Hirata, M. et al. Overview of BioBank Japan follow-up data in 32 diseases. J. Epidemiol. 27, S22-S28 (2017).

14. Loh, P.-R., Kichaev, G., Gazal, S., Schoech, A. P. \& Price, A. L. Mixed model association for biobank-scale data sets. Nat. Genet. 50, 906-908 (2018).

15. Benonisdottir, S. et al. Sequence variants associating with urinary biomarkers. Hum. Mol. Genet. 28, 1199-1211 (2019).

16. Gudbjartsson, D. F. et al. Association of variants at UMOD with chronic kidney disease and kidney stones-role of age and comorbid diseases. PLoS Genet. 6, e1001039-e1001039 (2010).

17. Amendola, L. M. et al. Actionable exomic incidental findings in 6503 participants: challenges of variant classification. Genome Res. 25, 305-315 (2015).
18. Boyle, A. P. et al. Annotation of functional variation in personal genomes using RegulomeDB. Genome Res. 22, 1790-1797 (2012).

19. Watanabe, K., Taskesen, E., Bochoven, A. \& Posthuma, D. Functional mapping and annotation of genetic associations with FUMA. Nat. Commun. https://doi.org/10.1038/s41467-017-01261-5 (2017).

20. de Leeuw, C. A., Mooij, J. M., Heskes, T. \& Posthuma, D. MAGMA: generalized gene-set analysis of GWAS data. PLoS Comput. Biol. 11, e1004219 (2015).

21. Schlingmann, K. P. et al. Mutations in CYP24A1 and idiopathic infantile hypercalcemia. N. Engl. J. Med. 365, 410-421 (2011).

22. Curhan, G. C., Willett, W. C., Speizer, F. E. \& Stampfer, M. J. Twentyfour-hour urine chemistries and the risk of kidney stones among women and men. Kidney Int. 59, 2290-2298 (2001).

23. Schlam, D. \& Canton, J. Every day I'm rufflin': calcium sensing and actin dynamics in the growth factor-independent membrane ruffling of professional phagocytes. Small GTPases 8, 65-70 (2017).

24. Gorvin, C. M. et al. AP2 mutations impair calcium-sensing receptor trafficking and signaling, and show an endosomal pathway to spatially direct G-protein selectivity. Cell Rep. 22, 1054-1066 (2018).

25. Wang, S.-K. et al. Critical roles for WDR72 in calcium transport and matrix protein removal during enamel maturation. Mol. Genet. Genom. Med. 3, 302-319 (2015).

26. Shang, G. et al. Structure analyses reveal a regulated oligomerization mechanism of the PlexinD1/GIPC/myosin VI complex. Elife 6, 213 (2017).

27. Nesbit, M. A. et al. Mutations in AP2S1 cause familial hypocalciuric hypercalcemia type 3. Nat. Genet. 45, 93-97 (2012).

28. Smith, K. R., Rajgor, D. \& Hanley, J. G. Differential regulation of the Racl GTPase-activating protein (GAP) BCR during oxygen/glucose deprivation in hippocampal and cortical neurons. J. Biol. Chem. 292, 20173-20183 (2017).

29. Nesbit, M. A. et al. Mutations affecting G-protein subunit $\alpha 11$ in hypercalcemia and hypocalcemia. N. Engl. J. Med. 368, 2476-2486 (2013).

30. Piret, S. E. et al. Identification of a G-protein subunit- $\alpha 11$ gain-of-function mutation, Val340Met, in a family with autosomal dominant hypocalcemia type 2 (ADH2). J. Bone Min. Res 31, 1207-1214 (2016).

31. Pearce, S. H. et al. A familial syndrome of hypocalcemia with hypercalciuria due to mutations in the calcium-sensing receptor. N. Engl. J. Med. 335, 1115-1122 (1996)

32. Gorvin, C. M. et al. Ga11 mutation in mice causes hypocalcemia rectifiable by calcilytic therapy. JCI Insight 2, e91103 (2017).

33. Sayers, J. et al. Successful treatment of hypercalcaemia associated with a CYP24A1 mutation with fluconazole. Clin. Kidney J. 8, 453-455 (2015).

34. Hannan, F. M., Babinsky, V. N. \& Thakker, R. V. Disorders of the calciumsensing receptor and partner proteins: insights into the molecular basis of calcium homeostasis. J. Mol. Endocrinol. 57, R127-R142 (2016).

35. Hannan, F. M. et al. The calcilytic agent NPS 2143 rectifies hypocalcemia in a mouse model with an activating calcium-sensing receptor (CaSR) mutation: relevance to autosomal dominant hypocalcemia type 1 (ADH1). Endocrinology 156, 3114-3121 (2015).

36. Babinsky, V. N. et al. Mutant mice with calcium-sensing receptor activation have hyperglycemia that is rectified by calcilytic therapy. Endocrinology 158, 2486-2502 (2017)

37. Sudlow, C. et al. UK biobank: an open access resource for identifying the causes of a wide range of complex diseases of middle and old age. PLoS Med 12, e1001779 (2015).

38. Tsugane, S. \& Sobue, T. Baseline Survey of JPHC study design and participation rate. J. Epidemiol. 11, 24-29 (2001).

39. Hamajima, N., . J-MICC Study Group. The Japan Multi-Institutional Collaborative Cohort Study (J-MICC Study) to detect gene-environment interactions for cancer. Asian Pac. J. Cancer Prev. 8, 317-323 (2007).

40. Kuriyama, S. et al. The Tohoku medical megabank project: design and mission. J. Epidemiol. 26, 493-511 (2016).

41. Bycroft, C. et al. The UK Biobank resource with deep phenotyping and genomic data. Nature 562, 203-209 (2018).

42. Akiyama, M. et al. Genome-wide association study identifies 112 new loci for body mass index in the Japanese population. Nat. Genet. 49, 1458-1467 (2017).

43. Tanikawa, C. et al. GWAS identifies two novel colorectal cancer loci at 16q24.1 and 20q13.12. Carcinogenesis 39, 652-660 (2018).

44. Purcell, S. et al. PLINK: a tool set for whole-genome association and population-based linkage analyses. Am. J. Hum. Genet. 81, 559-575 (2007).

45. The 1000 Genomes Project Consortium. et al. A global reference for human genetic variation. Nature 526, 68-74 (2015).

46. Abraham, G. \& Inouye, M. Fast principal component analysis of large-scale genome-wide data. PLoS ONE 9, e93766-5 (2014).

47. Consortium, T. I. H. 3 et al. Integrating common and rare genetic variation in diverse human populations. Nature 467, 52-58 (2010).

48. O’Connell, J. et al. Haplotype estimation for biobank-scale data sets. Nat. Genet. 48, 817-820 (2016). 
49. The Haplotype Reference Consortium. A reference panel of 64,976 haplotypes for genotype imputation. Nat. Genet. 48, 1279-1283 (2016).

50. Li, Y., Willer, C. J., Ding, J., Scheet, P. \& Abecasis, G. R. MaCH: using sequence and genotype data to estimate haplotypes and unobserved genotypes. Genet. Epidemiol. 34, 816-834 (2010).

51. Loh, P.-R. et al. Efficient Bayesian mixed-model analysis increases association power in large cohorts. Nat. Genet. 47, 284-290 (2015).

52. Cai, Q. et al. Genome-wide association analysis in East Asians identifies breast cancer susceptibility loci at 1q32.1,5q14.3 and 15q26.1. Nat. Genet. 46, 886-890 (2014).

53. Mägi, R. \& Morris, A. P. GWAMA: software for genome-wide association meta-analysis. BMC Bioinf. 11, 288 (2010).

54. Wang, K., Li, M. \& Hakonarson, H. ANNOVAR: functional annotation of genetic variants from high-throughput sequencing data. Nucleic Acids Res. 38, e164-e164 (2010).

55. Battle, A., Brown, C. D., Engelhardt, B. E. \& Montgomery, S. B. Genetic effects on gene expression across human tissues. Nature 550, 204-213 (2017).

56. Babinsky, V. N. et al. Allosteric modulation of the calcium-sensing receptor rectifies signaling abnormalities associated with G-protein $\alpha-11$ mutations causing hypercalcemic and hypocalcemic disorders. J. Biol. Chem. 291, 10876-10885 (2016)

57. Leach, K. et al. Impact of clinically relevant mutations on the pharmacoregulation and signaling bias of the calcium-sensing receptor by positive and negative allosteric modulators. Endocrinology 154, 1105-1116 (2013).

58. Gorvin, C. M. et al. Cinacalcet rectifies hypercalcemia in a patient with familial hypocalciuric hypercalcemia type 2 (FHH2) caused by a germline loss-offunction Ga11 mutation. J. Bone Min. Res 33, 32-41 (2018).

59. Cuzick, J. A Wilcoxon-type test for trend. Stat. Med. 4, 87-90 (1985).

60. StataCorp. Stata 15 Base Reference Manual. College Station, TX: Stata Press (2017).

\section{Acknowledgements}

This work was supported by grants from Kidney Research UK (RP_030_20180306) to S. A.H., A.W., M.G., B.W.T., and D.F, National Institute for Health Research (N.I.H.R) Oxford Biomedical Research Centre to R.V.T, and the Wellcome Trust (204826/z/16/z) to S.A.H, and M.G. S.A.H. is a N.I.H.R Academic Clinical Lecturer. A.W is an MRC Clinical Research Training Fellow. R.V.T. has Senior Investigator Awards from the Wellcome Trust (106995/z/15/z) and N.I.H.R. (NF-SI-0514-10091). We acknowledge the contribution to this study made by the Oxford Centre for Histopathology Research and the Oxford Radcliffe Biobank, which are supported by the NIHR Oxford Biomedical Research Centre.

\section{Author contributions}

S.A.H., A.W., B.W.T. and D.F. designed this study. S.A.H., A.W., M.G., E.G., C.Ta., Y.K. C.Te., A.T., M.K., K.M. and B.W.T. acquired data. A.W. and D.F. carried out genetic association analysis. S.A.H., M.G., A.L.B., and A.K.G. undertook in vitro studies. S.A.H., A.W., M.G., M.N., C.Ta., Y.K., C.Te., A.T., M.K., K.M., R.V.T, B.W.T. and D.F. analyzed and interpreted data. S.A.H., A.W., R.V.T., B.W.T. and D.F. wrote the first draft of the manuscript. All other co-authors participated in the preparation of the manuscript by reading and commenting on the draft prior to submission.

\section{Competing interests}

The authors declare no competing interests.

\section{Additional information}

Supplementary information is available for this paper at https://doi.org/10.1038/s41467019-13145-x.

Correspondence and requests for materials should be addressed to S.A.H.

Peer review information Nature Communications thanks Bidhan Bandyopadhyay and other, anonymous, reviewers for their contributions to the peer review report of this work. Peer review reports are available.

Reprints and permission information is available at http://www.nature.com/reprints

Publisher's note Springer Nature remains neutral with regard to jurisdictional claims in published maps and institutional affiliations.

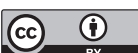

Open Access This article is licensed under a Creative Commons Attribution 4.0 International License, which permits use, sharing, adaptation, distribution and reproduction in any medium or format, as long as you give appropriate credit to the original author(s) and the source, provide a link to the Creative Commons license, and indicate if changes were made. The images or other third party material in this article are included in the article's Creative Commons license, unless indicated otherwise in a credit line to the material. If material is not included in the article's Creative Commons license and your intended use is not permitted by statutory regulation or exceeds the permitted use, you will need to obtain permission directly from the copyright holder. To view a copy of this license, visit http://creativecommons.org/ licenses/by/4.0/.

(c) The Author(s) 2019, corrected publication 2022 